\title{
Effects of biological manganese cycling on carbon storage in the critical zone
}

\author{
ELIZABETH HERNDON ${ }^{1}$, SYDNEY LAUBSCHER ${ }^{2}$, \\ BENJAMIN SULMAN ${ }^{3}$, CARLA ROSENFELD ${ }^{4}$
}

${ }^{1}$ Environmental Sciences Division, Oak Ridge National Laboratory; Oak Ridge TN, USA 37831;

(herndonem@ornl.gov)

${ }^{2}$ Department of Geology, Kent State University, Kent OH, USA, 44240

${ }^{3}$ Environmental Sciences Division, ORNL; Oak Ridge TN, USA 37831

${ }^{4}$ Quantitative Bio-element Imaging Center, Northwestern University, Evanston IL, USA 60208

Manganese (Mn) cycling between soils and plants has the potential to influence carbon dynamics in the critical zone by modifying rates of litter decomposition and by generating reactive Mn oxides that either stabilize or degrade organic compounds. However, the extent to which these processes regulate carbon storage remains unknown. Here, we couple results from a greenhouse experiment with process-based modeling to explore the hypothesis that increases in $\mathrm{Mn}$ cycling through terrestrial ecosystems drive decreases in carbon storage. First, red maple saplings grown in synthetic media were supplied with either aqueous or mineral-hosted $\mathrm{Mn}$ to evaluate how mineral dissolution kinetics regulate rates of plant uptake. High throughput rRNA gene sequencing (16S and ITS1) was completed on bulk and rhizosphere soils to investigate changes in bacterial and fungal community composition in response to Mn additions. Second, a coupled element cycling model was developed in PFLOTRAN to investigate how $\mathrm{Mn}$ enrichment influences soil $\mathrm{C}$ storage through processes including lignin degradation and mineral sorption. We determined that Mn uptake varied as a function of mineral dissolution kinetics but was tempered by biological processes; that is, increases in Mn uptake were moderate relative to increases in Mn solublity. Potential bacterial and fungal Mn-oxidizers were detected in all treatments and were higher in planted than soil-only pots, signifying that microbial $\mathrm{Mn}$ oxidation may also limit $\mathrm{Mn}$ solubility in soils. Initial modeling results indicate that high concentrations of available $\mathrm{Mn}^{2+}$ accelerate lignin oxidation and conversion to more readily biodegradable substates, ultimately limiting soil $\mathrm{C}$ storage. This work provides preliminary evidence that biogeochemical Mn cycling has a potentially pronounced impact on $\mathrm{C}$ cycling in the critical zone. Future work aims to better constrain model outputs through experimental validation of modeling parameters. 